\title{
EL VOLUMEN CORPUSCULAR MEDIO ALTO SE ASOCIA CON UN AUMENTO DE LOS GLÓBULOS BLANCOS EN EL EMBARAZO
}

\author{
Cinthya Vásquez-Velásquez¹, Diego Fano', Luis Aguilar Cruces², José Luis López Cuba², \\ Tania Paredes Quiliche², Juana Molina², Gustavo F. Gonzales'.
}

\begin{abstract}
RESUMEN
Objetivos. Determinar si el volumen corpuscular medio (VCM) alto se relaciona con el recuento de glóbulos blancos (GB) en gestantes atendidas en el Instituto Nacional Materno Perinatal. Materiales y métodos. Estudio observacional transversal, compuesto por 8590 hemogramas obtenidos de gestantes atendidas en el Instituto Nacional Materno Perinatal durante el segundo semestre del 2018. Se utilizó el equipo automatizado CELL-DYN Ruby ®. Para el análisis estadístico, se usó el paquete estadístico STATA versión 15.0. Los datos son presentados como medias \pm error estándar. La variable VCM se categorizó como normal $(80 \leq \mathrm{MCV} \leq 97 \mathrm{fL})$, baja (MCV <80fL) y alta (MCV> $97 f \mathrm{~L})$. La prueba ANOVA se empleó para evaluar la diferencia entre los grupos de VCM y los diversos parámetros hematológicos; en caso de resultar significativo, se empleó la prueba post-hoc de Scheffé. La prueba de regresión lineal se realizó para determinar el coeficiente de los grupos de VCM y su variación con el recuento de Glóbulos Blancos (GB). Resultados. El VCM alto en gestantes se asocia a un aumento de GB. En el análisis de cada tipo de célula blanca, se observan que todas ellas, con excepción de los neutrófilos, se encuentran elevadas cuando VCM es categorizado como alto. Las plaquetas muestran un valor menor en la categoría de VCM alto a diferencia de lo observado en las categorías de VCM normal y bajo. Conclusiónes. EI VCM elevado parece asociarse a un proceso inflamatorio, evidenciado en un aumento de la serie blanca con excepción de los neutrófilos.
\end{abstract}

Palabras claves: Hemograma Automatizado, Volumen Corpuscular Medio, Glóbulos Blancos, Gestación, inflamación (Fuente: DeCS BIREME).

\section{HIGH MEAN CORPUSCULAR VOLUME IS ASSOCIATED WITH AN INCREASE OF WHITE BLOOD CELLS IN PREGNANCY}

\begin{abstract}
Introduction. During pregnancy, several changes occur in the hematological parameters. The computerized blood count is one of the most useful tools to analyze them, being recommended by the World Health Organization for anemia diagnosis. However, not all the parameters obtained are employed in the clinical practice for the diagnosis of different pathologies or alterations during pregnancy. Objectives. To determine if the mean corpuscular volume (MCV) is related with the white blood cell (GB) count in pregnant women who attend to their regular controls at the National Maternal Perinatal Institute. Materials and methods. Observational cross-sectional study, composed of 8590 pregnant women attended in the National Maternal Perinatal Institute in Lima. An automated analysis was performed with the Mindray BC5800 25-parameter equipment. Statistical software STATA version 15.0 was used. The data are presented as means \pm standard error, the variable VCM was categorized as normal $(80 \leq \mathrm{MCV} \leq 97 \mathrm{fL})$, low (MCV <80fL) and high (MCV > $97 \mathrm{fL})$. The ANOVA test was performed to determine the statistical difference between the MCV categorization groups and the various hematological parameters, followed by the post-hoc Scheffé test if significant. Linear regression was performed to determine the coefficient of the MCV categorization groups and their variation with the White Blood Cell count. Results. High MCV in pregnant women is associated with an increase of GB. In the analysis of the various types of GB, all of them are increased except for neutrophils, when MCV is high. Platelets show a lower value in the high MCV category as opposed to the normal and low VCM categories. Conclusions. High MCV could be associated with inflammation, as evidenced in the high number of the different GB except for neutrophils.
\end{abstract}

Key words: Automated Blood Count, Mean Corpuscular Volume, White Blood Cells, Pregnant women, Inflammation (Source: MeSH NLM).

\footnotetext{
Laboratorio de Endocrinología y Reproducción. Facultad de Ciencias y Filosofía, e Instituto de Investigaciones de la Altura. Universidad Peruana Cayetano Heredia.

2 Instituto Nacional Materno Perinatal. Lima, Perú.

Citar como: Vásquez-Velásquez C, Fano D, Aguilar L, Lopez JL, Paredes T, Molina J, Gonzales GF. El Volumen Corpuscular Medio alto se asocia con un aumento de los glóbulos blancos en el embarazo. Rev Peru Investig Matern Perinat 2019; 8(3):10-6 DOI https://doi.org/10.33421/inmp.2019159
} 


\section{INTRODUCCIÓN}

La gestación es un proceso de cambios inmunológicos que permiten al embrión poder implantarse, crecer y desarrollarse adecuadamente 1 . De igual manera, se producen variaciones a nivel hematológico, siendo estos cruciales, ya que tienen la finalidad de lograr satisfacer las demandas del feto en desarrollo y de la placenta ${ }^{2}$.

Se sabe que una de las demandas en la gestación es el hierro, cuyo requerimiento aumenta en un gramo. Como consecuencia, la hepcidina, hormona hepática de composición proteica, es inhibida. Al ser bloqueada su transcripción, se genera un aumento en la biodisponibilidad de hierro para aportar al proceso eritropoyético, aumentando con ello, la masa celular roja. Este cambio debe ir acompañado de un aumento del volumen plasmático (aumento de un 40-45\% aproximadamente $)^{3}$, reduciendo así la concentración de hemoglobina debido al proceso de hemodilución, de tal forma que el flujo útero-placentario no sé afecte por la mayor masa hemática, y se garantice un correcto transporte y entrega de nutrientes para la supervivencia, crecimiento y desarrollo del feto. El cambio del volumen plasmático es mediado hormonalmente, y está asociado a la acción de los estrógenos y de la progesterona sobre el riñón, favoreciendo con esto el aumento en el contenido total de agua corporal ${ }^{4-6}$.

Se considera que el embarazo constituye un proceso inflamatorio. Por ello, otro de los cambios hematológicos importantes es el aumento de los parámetros leucocitarios en respuesta al estrés fisiológico inducido por el embarazo, y una disminución en el conteo de plaquetas debido a la hemodilución ${ }^{7-10}$. Es importante tener en cuenta que el conteo de glóbulos blancos por unidad de volumen incrementa a pesar de la hemodilución, lo que indica que efectivamente hay un aumento en la producción de la serie blanca.

La disminución de la concentración de la hemoglobina por la hemodilución genera una "anemia fisiológica" que por sí misma no es dañina, sino más bien beneficiosa ${ }^{11,12}$. De aquí nace la pregunta, ¿cuándo se considera que un valor bajo de la concentración de hemoglobina es realmente una anemia y no una "hemodilución"? Ciertamente una medición sola de la hemoglobina no permite determinar las diferencias entre una hemodilución de una verdadera anemia

Por ello, en el 2016, la Organización Mundial de la Salud $(\mathrm{OMS})^{13}$ en su reporte de cuidados prenatales indica la importancia de realizar análisis del hemograma automatizado completo, ya que este permite tener un panorama mucho más amplio de la situación hematológica de la gestante y el feto. En el Perú, a pesar de que el análisis de los parámetros hematológicos usando multianalizadores automáticos se ha incrementado, poco o nada se ha publicado sobre las diferentes variables hematológicas que resultan de su medición. Es más, no todos los parámetros que se obtienen como resultados de una evaluación hematológica son usados en la rutina clínica.

Uno de los parámetros que han mostrado una gran utilidad en evaluar el impacto de la hemoglobina sobre el resultado del embarazo y del recién nacido, es el volumen corpuscular medio (VCM). Steer ${ }^{14}$ mostró que valores de concentración de hemoglobina en el rango de anemia leve produce los mejores resultados del embarazo cuando el VCM es > 84 fL. Esto resalta la importancia de su medición.

Se estima que valores bajos de VCM junto con valores bajos de hemoglobina, diagnostican a una anemia ferropénica microcítica. Cuando el VCM es $>97 \mathrm{fL}$ en presencia de anemia ${ }^{15}$, se considera como anemia megaloblástica, y ha sido relacionada a la deficiencia de folato, y de vitamina B12.

Hay estudios, sin embargo, que demuestran la presencia de VCM elevado sin anemia ${ }^{15}$, los cuales estarían relacionados con enfermedades inflamatorias ${ }^{16}$.

Por ello, el presente estudio trata de determinar si uno de los parámetros hematológicos, que actualmente sirven para el diagnóstico de anemia macrocítica y microcítica, el Volumen Corpuscular Medio, estaría relacionado con el recuento de glóbulos blancos en gestantes atendidas en el Instituto Nacional Materno Perinatal.

\section{MATERIALES Y MÉTODOS}

Diseño del estudio: Estudio de corte transversal que consistió en el análisis de una base de datos obtenida del Laboratorio de Hematología del Instituto Nacional Materno Perinatal. Se analizaron un total de 8590 hemogramas utilizando el multianalizador automático CELL-DYN Ruby ${ }^{\circledR}$ de 25 parámetros. Los parámetros hematológicos corresponden al análisis rutinario realizado a las mujeres embarazadas atendidas en el Instituto Nacional Materno Perinatal correspondiente al segundo semestre del 2018.

Variables hematológicas: El multianalizador automático CELL-DYN Ruby \& otorga un total de 25 parámetros hematológicos. Se caracteriza porque su método de detección es basado en láser óptico usando la tecnología MAPSS (Multi Angle Polarized Scatter Separation).

Del total de parámetros hematológicos analizados solo se consideraron las siguientes para el estudio: volúmenes corpusculares (Volumen Corpuscular Medio, Distribución de ancho del Eritrocito), plaquetas, masa leucocitaria (linfocitos, neutrófilos, monocitos, eosinófilos y basófilos), hemoglobina, hematocrito, Hemoglobina Corpuscular Media y Conteo de Células Rojas. 
Las unidades usadas para: los tipos de células (glóbulos blancos, glóbulos rojos y plaquetas) fueron medidas por conteo total $\left({ }^{*} 10^{9}\right), \mathrm{g} / \mathrm{dL}$ para hemoglobina, \% para hematocrito y RDW, fL para VCM y pg/célula para Hemoglobina Corpuscular Media.

El volumen corpuscular medio fue categorizado como normal $(80 \leq \mathrm{VCM} \leq 97 \mathrm{fl})$, baja (VCM <80fl) y alta $(\mathrm{VCM}>$ $97 \mathrm{fl})^{15}$.

\section{Control de calidad}

A manera de control de calidad, cada tres días se agregan muestras de valor conocido proporcionados por el proveedor y que corresponden a valores hematológicos bajo, normal o alto para los siguientes parámetros: glóbulos blancos (linfocitos, neutrófilos, monocitos, eosinófilos y basófilos), glóbulos rojos, plaquetas, hemoglobina y VCM. En el caso de la diferenciación de la carga leucocitaria, se consideró el conteo total y el porcentaje.

Se sabe por la literatura que la tasa de falsos negativos es de $3.4 \%$ y la tasa de falsos positivos es de $17 \%$ en el análisis correspondiente al equipo CELL-DYN Ruby ${ }^{\circledR}{ }^{17}$.

A continuación, se presentan los valores de cada parámetro hematológico evaluado correspondiente a los 3 niveles de control de calidad (bajo, normal y alto), los cuales sirven para estandarizar y controlar la masa celular blanca:
Consideraciones éticas: El trabajo contó con la aprobación del comité institucional de ética del INMP (expediente número: INMP 18-14572-1) y registro en la Universidad Peruana Cayetano Heredia (código SIDISI: 101555). Los datos fueron manejados mediante códigos para salvaguardar la información confidencial de las gestantes.

Base de datos: El número total de observaciones de la base de hemogramas del segundo semestre del 2018 era de 10000 mediciones. Posterior a la limpieza de datos, fueron consideradas 8591 observaciones.

En la limpieza se descartaron un total de 1409 observaciones:

- 665 datos correspondientes a las corridas background de cada uno de los días que se realizan hemogramas, estas se distinguían, debido que no tenían valores hematológicos.

- 138 observaciones correspondientes a los controles (bajo, normal y alto), medidos cada 3 días de análisis.

- 578 valores aberrantes, los cuales eran fácilmente distinguibles, puesto que los valores hematológicos presentaban asteriscos.

- 58 observaciones, las cuales eran valores duplicados de hemogramas, y fueron eliminadas para evitar sobrestimación de cualquiera de las categorías.

Análisis estadístico: Para el análisis estadístico, se utilizó el paquete estadístico STATA versión 15.0 para

Tabla 1. Parámetros leucocitarios y plaquetas correspondientes a los controles de calidad

\begin{tabular}{|c|c|c|c|c|c|c|c|c|}
\hline Control & $\mathbf{N}$ & $\begin{array}{l}\text { Glóbulos blancos } \\
\left(\times 10^{9}\right)(\text { media } \pm e . e)\end{array}$ & $\begin{array}{c}\text { Monocitos } \\
\left(\times 10^{\circ}\right) \\
\text { (mediate.e) }\end{array}$ & $\begin{array}{c}\text { Linfocitos } \\
\left(\times 10^{9}\right) \\
\text { (media } \pm \text { e.e) }\end{array}$ & $\begin{array}{l}\text { Neutrófilos } \\
\left(\times 10^{9}\right) \\
\text { (media } \pm \text { e.e) }\end{array}$ & $\begin{array}{c}\text { Eosinófilos } \\
\left(\times 10^{9}\right) \\
(\text { media } \pm \text { e.e) }\end{array}$ & $\begin{array}{c}\text { Basófilos } \\
\left(\times 10^{9}\right) \\
\text { (mediate.e) }\end{array}$ & $\begin{array}{l}\text { Plaquetas } \\
\left(\times 10^{9}\right) \\
\text { (media } \pm \text { e.e) }\end{array}$ \\
\hline Bajo & 47 & $3.78 \pm 0.037$ & $0.23 \pm 0.024$ & $1.10 \pm 0.031$ & $2.24 \pm 0.012$ & $0.107 \pm 0.0023$ & $0.096 \pm 0.005$ & $79.12 \pm 0.79$ \\
\hline Normal & 45 & $7.11 \pm 0.070$ & $0.48 \pm 0.043$ & $2.09 \pm 0.063$ & $4.19 \pm 0.021$ & $0.19 \pm 0.0036$ & $0.15 \pm 0.009$ & $211.15 \pm 1.05$ \\
\hline Alto & 46 & $16.62 \pm 0.045$ & $1.51 \pm 0.012$ & $4.58 \pm 0.062$ & $9.67 \pm 0.046$ & $0.44 \pm 0.0067$ & $0.40 \pm 0.011$ & $471.65 \pm 1.49$ \\
\hline
\end{tabular}

Los datos son mediasterror estándar

Tabla 2. Parámetros hematológicos correspondientes a los controles de calidad

\begin{tabular}{|c|c|c|c|c|c|c|c|}
\hline Control & $\mathbf{N}$ & $\begin{array}{c}\mathrm{Hb}(\mathrm{g} / \mathrm{dL}) \\
\text { (mediate.e) }\end{array}$ & $\begin{array}{c}\text { Hcto (\%) } \\
\text { (media } \pm \text { e.e) }\end{array}$ & $\begin{array}{c}\text { VCM (fL) } \\
\text { (media士e.e) }\end{array}$ & $\begin{array}{l}\mathrm{RBC}\left(\mathrm{x} 10^{9}\right) \\
\text { (mediate.e) }\end{array}$ & $\begin{array}{c}\mathrm{HCM}(\mathrm{pg} / \\
\text { célula) } \\
\text { (media士e.e) }\end{array}$ & 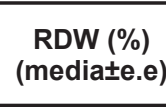 \\
\hline Bajo & 47 & $7.10 \pm 0.010$ & $19.95 \pm 0.15$ & $69.16 \pm 0.52$ & $2.88 \pm 0.0069$ & $24.63 \pm 0.048$ & $14.56 \pm 0.090$ \\
\hline Normal & 45 & $11.80 \pm 0.010$ & $32.18 \pm 0.23$ & $73.92 \pm 0.55$ & $4.35 \pm 0.009$ & $27.11 \pm 0.051$ & $13.35 \pm 0.079$ \\
\hline Alto & 46 & $15.78 \pm 0.10$ & $41.78 \pm 0.25$ & $78.00 \pm 0.58$ & $5.36 \pm 0.015$ & $29.00 \pm 0.081$ & $12.15 \pm 0.167$ \\
\hline
\end{tabular}

Los datos son medias \pm error estandar. 
computadoras personales (Stata Corporation, 4905 Lakeway Drive, College Station, TX 77845, USA). Los datos son presentados como medias \pm error estándar. La prueba ANOVA determinó la diferencia estadística entre los grupos de VCM y los diversos parámetros hematológicos, la prueba Scheffé como post-hoc. La prueba de regresión lineal se realizó para determinar el coeficiente de regresión los grupos de VCM y su variación con el recuento de Glóbulos Blancos (GB).

\section{RESULTADOS}

El conteo medio de glóbulos blancos muestra un valor medio más elevado en el grupo de VCM alto, que las otras dos categorías. Además de ello, este valor es estadísticamente significativo frente al grupo normal. De similar manera, la Figura 1 muestra que existe una asociación positiva entre el aumento de VCM y el conteo de glóbulos blancos, teniendo un $r^{2}=0.4729$ y un $r=0.687$.

Al comparar el conteo de los distintos tipos de glóbulos blancos se observa que el grupo con VCM Bajo, si bien no muestra una diferencia en el conteo total de GB con el grupo de VCM normal (Tabla 3), el número de linfocitos es mayor a comparación del grupo con VCM normal (Tabla 4). En cuanto al VCM Alto, este muestra un mayor conteo de las distintas células a excepción de los neutrófilos comparado con el VCM normal y bajo; mientras que, en el caso de las plaquetas, el grupo con VCM bajo presenta una mayor concentración de plaquetas. Contrariamente, el grupo con VCM alto posee el menor conteo de plaquetas comparados con los grupos de VCM normal y VCM bajo (Tabla 4).

En la tabla 5 se puede evidenciar que el grupo con VCM alto muestra un mayor valor de $\mathrm{Hb}$, Hto y $\mathrm{HCM}(11.91 \mathrm{~g} /$ dL, $37.10 \%$ y 32.42 pg) a comparación de los observados
Tabla 3. Regresión lineal entre las categorías de conteo de VCM y Glóbulos blancos (GB).

\begin{tabular}{lcc}
\hline Categorías VCM & $\mathbf{N}$ & GB(media \pm e.e) $\times 10^{9}$ \\
\hline Normal & 6563 & $9.41 \pm 0.039$ \\
Bajo & 862 & $9.47 \pm 0.12$ \\
Alto & 1166 & $9.81 \pm 0.12^{*}$ \\
\hline
\end{tabular}

Los datos son medias \pm error estandar* $p<0.0001$ con respecto a los valores en el grupo de VCM normal.

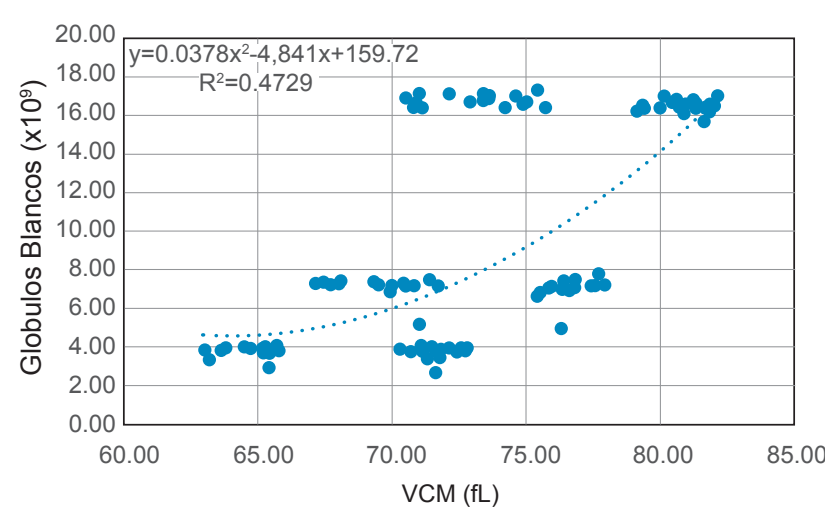

Figura 1. Regresión lineal entre VCM (fL) vs Conteo de globulos blancos.

en los grupos con VCM Normal y Bajo; sin embargo, para el RBC (3.70 células/mcL) este resulta ser menor, y el RDW (13.28\%) es similar que el grupo Normal y menor frente al Bajo VCM. Resultados inversos se observan con Bajo VCM, puesto que presenta los menores valores para $\mathrm{Hb}$, Hto y HCM, mientras que para RBC y RDW es mayor (4.24 células/mcLy $16.55 \%$ respectivamente).

Tabla 4. Medias \pm error estándar de los distintos tipos de glóbulos blancos y plaquetas con respecto a las categorías de VCM.

\begin{tabular}{|c|c|c|c|c|c|c|c|}
\hline $\begin{array}{l}\text { Categoría } \\
\text { VCM }\end{array}$ & $\mathbf{N}$ & $\begin{array}{c}\text { Monocitos }\left(\times 10^{9}\right) \\
(\text { media } \pm \text { e.e })\end{array}$ & $\begin{array}{l}\text { Linfocitos }\left(\times 10^{9}\right) \\
\text { (media } \pm \text { e.e) }\end{array}$ & $\begin{array}{c}\text { Neutrófilos }\left(\times 10^{9}\right) \\
(\text { media } \pm \text { e.e })\end{array}$ & 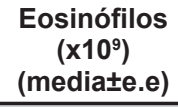 & $\begin{array}{c}\text { Basófilos }\left(\times 10^{9}\right) \\
\text { (mediate.e) }\end{array}$ & $\begin{array}{l}\text { Plaquetas }\left(\times 10^{9}\right) \\
\text { (media } \pm \text { e.e) }\end{array}$ \\
\hline Bajo & 862 & $0.592 \pm 0.008$ & & & $0.206 \pm 0.007$ & $0.08 \pm 0.0011$ & $287.51 \pm 3.09^{*}$ \\
\hline Normal & 6563 & $0.575 \pm 0.002$ & $2.111 \pm 0.009$ & $6.442 \pm 0.038$ & $0.212 \pm 0.003$ & $0.07 \pm 0.0004$ & $252.07 \pm 0.98$ \\
\hline Alto & 1166 & $0.710 \pm 0.015^{* a}$ & $2.372 \pm 0.057^{*}, \mathrm{a}$ & $6.43 \pm 0.100$ & $0.230 \pm 0.009^{*}$ & $0.10 \pm 0.005^{*, a}$ & $240.90 \pm 2.64 * a$ \\
\hline
\end{tabular}

Test ANOVA: * $p<0.05$ con respecto a los valores en el grupo de VCM normal. a $p<0.05$ con respecto a los valores en el grupo de VCM bajo.

Tabla 5. Medias \pm error estándar de Hemoglobina ( $\mathrm{Hb})$, Hematocrito (Hto), Hemoglobina Corpuscular Media (HCM), Conteo de Glóbulos Rojos (RBC), Ancho de la distribución de Glóbulo rojo (RDW) con respecto a las categorías de VCM.

\begin{tabular}{|c|c|c|c|c|c|c|}
\hline $\begin{array}{l}\text { Categorías } \\
\text { VCM }\end{array}$ & $\mathbf{N}$ & $\begin{array}{c}\mathrm{Hb}(\mathrm{g} / \mathrm{dL} \text { ) } \\
\text { (mediate.e) }\end{array}$ & $\begin{array}{c}\text { Hto (\%) } \\
\text { (media } \pm \text { e.e) }\end{array}$ & 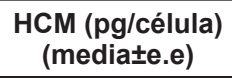 & 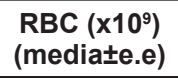 & $\begin{array}{c}\text { RDW (\%) } \\
\text { (media } \pm \text { e.e) }\end{array}$ \\
\hline Bajo & 862 & $9.96 \pm 0.06 *$ & $31.32 \pm 0.153^{*}$ & $23.57 \pm 0.12^{*}$ & $4.24 \pm 0.02^{*}$ & $16.55 \pm 0.11^{*}$ \\
\hline Normal & 6563 & $11.61 \pm 0.018$ & $36.20 \pm 0.057$ & $28.96 \pm 0.02$ & $4.02 \pm 0.006$ & $13.30 \pm 0.02$ \\
\hline Alto & 1166 & $11.91 \pm 0.04^{*}, \mathrm{a}$ & $37.10 \pm 0.139^{*}$ & $32.42 \pm 0.17^{*}, \mathrm{a}$ & $3.70 \pm 0.01^{* a}$ & $13.28 \pm 0.06^{a}$ \\
\hline
\end{tabular}

Test ANOVA: * $p<0.05$ con respecto a los valores en el grupo de VCM normal. ${ }^{a} p<0.05$ con respecto a los valores en el grupo de VCM bajo. 


\section{DISCUSIÓN}

En la gestación, se deben de presentar cambios hematológicos para satisfacer las nuevas demandas de la gestante y el feto, y así propiciar un correcto crecimiento, desarrollo y diferenciación de los componentes materno-fetales ${ }^{18}$.

Algunos de los cambios hematológicos que ocurren en la gestación son un aumento de la masa leucocitaria ${ }^{19}$, disminución de plaquetas ${ }^{7,8}$, etc. Sin embargo, algunas de las alteraciones visualizadas en el hemograma automatizado pueden servir como indicadores indirectos de patologías.

Los valores bajos de VCM asociados con hipocromía (valores bajos de $\mathrm{HCM}$; $\leq 27 \mathrm{pg}$ ) se han relacionado con anemia por deficiencia de hierro ${ }^{20}$. También se le observa en la talasemia (es más frecuente en las beta-talasemias) o en la anemia sideroblástica; y en situaciones de intoxicación por plomo o cobre. En estos casos, el eritrocito no tiene la capacidad de transportar la cantidad necesaria de elementos (grupo hemo, hemoglobina, hierro y oxigeno) clave para la correcta fisiología de las gestantes y del feto. Por otro lado, el VCM alto se ha relacionado a anemia por deficiencia de folatos y desarrollo de procesos inflamatorios ${ }^{21}$.

En la tabla 3 podemos evidenciar que el proceso inflamatorio sería una causa de este incremento en el VCM. Se observa que todos los tipos de glóbulos blancos están aumentados en la categoría de VCM alto, siendo los neutrófilos los únicos que se mantienen en concentración normal; no obstante, estos al bajar progresivamente son un indicador de enfermedades inflamatorias crónicas ${ }^{22}$.

Por otro lado, el conteo de glóbulos rojos se encuentra bajo en el VCM alto. La explicación a esto es que en el campo de visión, debido al aumento de VCM, el número de eritrocitos será menor; sin embargo, esto se debió a un conteo de la maquina por una misma unidad de volumen en todas las muestras.

Domínguez Ruiz de León y col ${ }^{15}$ en un estudio realizado en población urbana encontró una prevalencia de $7.12 \%$ de macrocitosis sin anemia, a lo cual se determinó que se debía a un proceso inflamatorio como hipotiroidismo, alteraciones gástricas, condición de fumador, etc. En el análisis, se encontró que del total de gestantes con macrositosis, $21.7 \%$ presentaban anemia y un $78.3 \%$ no. Por lo que respalda que estos valores de masa celular blanca se encuentren elevados como consecuencia de un VCM alto.

Así mismo, en 1976 se evidenció que la suplementación de hierro en la gestación genera un aumento del $V_{C M}{ }^{21}$. En el Perú, como normativa obligatoria se distribuye y suplementa con hierro a las gestantes anémicas y no anémicas, sin generar cambios en las estadísticas actuales, lo que estaría evidenciando que la política supeditada no es efectiva ${ }^{23}$, y por el contrario, incrementaría las cifras debido que el proceso inflamatorio es la segunda principal causal de anemia en el mundo ${ }^{24}$.

El aumento de Interleucinas proinflamatorias genera la activación de la producción de hepcidina, hormona hepática que bloquea e internaliza a la ferroportina, disminuyendo la biodisponibilidad del hierro para la producción eritropoyética y por ende desencadenado anemia ${ }^{25,26}$

En la figura 1 se muestra el comportamiento de las categorías del VCM con respecto al conteo de glóbulos blancos, el cual es una característica curva en forma de "u" o "j". Este comportamiento se ha evidenciado en múltiples análisis con repercusión en la salud materna y perinatal ${ }^{12}$. Se evidencia que los valores extremos, categorías de VCM bajo ( $<80 \mathrm{fL})$ y VCM alto $(110 \mathrm{fL}$ ) presentan alteraciones en la masa leucocitaria, la cual tiene una función importante durante la gestación.

El determinar parámetros hematológicos comprendidos en hemogramas automatizados como predictores a condiciones fisiopatológicas es de crucial importancia, debido que el análisis de biomarcadores como Interleucinas proinflamatorias, Proteína C Reactiva, Glicoproteína ácida $1 \alpha$, etc. tiene un alto costo por muestra, mientras que el hemograma automatizado tiene un precio asequible y es medido en muchos centros de salud del país.

Si bien el presente estudio muestra como una de sus principales limitantes el no contar con análisis diferenciados por trimestre de gestación, debido a que la base de datos no indica información como la edad gestacional de la gestante al momento de la evaluación, permite revalorar el empleo del hemograma automatizado como una potencial herramienta para la detección de procesos inflamatorios que podrían afectar la salud de la madre como del feto.

En conclusión, se debe de recalcar la importancia de conocer los valores de normalidad de los parámetros hematológicos en poblaciones vulnerables como las gestantes, los cuales sirven como predictores de patologías y alteraciones que repercuten sobre los resultados maternos y perinatales. Así mismo, se debe implementar como política de las instituciones públicas el análisis del hemograma automatizado para tener un panorama amplio sobre la situación de las gestantes y acoplarnos a la recomendación de la Organización Mundial de la Salud (2016), la cual prioriza el uso del hemograma automatizado sobre el hemoglobinómetro ${ }^{13}$.

Agradecimientos: Los autores agradecen al personal Tecnólogo Médico del Laboratorio de Hematología 
del Instituto Materno Perinatal que brindo todas las facilidadespara la obtención de muestras y base de datos.

Financiamiento: Proyecto de Investigación Canon UNSAAC, código N-016-2018, titulado "La hemoglobina y la homeostasis de hierro en Lima $(150 \mathrm{~m})$ y Cusco (3400 m)".

Conflicto de interés: Los autores declaran no tener algún conflicto de intereses.

\section{REFERENCIAS BIBLIOGRÁFICAS}

1. Barañao RI. Immunology of pregnancy. Invest Clin [Internet]. 2011 Jun [cited 2019 May 23];52(2):175-94. Available from: http://www.ncbi.nlm.nih.gov/pubmed/21866790

2. Ojeda González JJ, Rodríguez Älvarez M, Estepa Pérez JL, Piña Loyola CN, Cabeza Poblet BL. Physiological Changes during Pregnancy. Its Relevance for the Anesthesiologist MediSur [Internet]. 2003 [cited 2019 May 23];9(5):484-91. Available from: http://scielo.sld.cu/scielo.php?script=sci_ arttext\&pid=S1727-897X2011000500011

3. Lund CJ, Donovan JC. Blood volume during pregnancy. Significance of plasma and red cell volumes. Am J Obstet Gynecol [Internet]. 1967 Jun 1 [cited 2019 Apr 26];98(3):394-403. Available from: http://www.ncbi.nlm. nih.gov/pubmed/5621454

4. Carlin A, Alfirevic Z. Physiological changes of pregnancy and monitoring. Best Pract Res Clin Obstet Gynaecol [Internet] 2008 Oct [cited 2019 May 23];22(5):801-23. Available from: http://www.ncbi.nlm.nih.gov/pubmed/18760680

5. Sharma R, Sharma S. Physiology, Blood Volume [Internet]. StatPearls. 2018 [cited 2019 May 23]. Available from: https://www.ncbi.nlm.nih.gov/pubmed/30252333

6. Costantine MM. Physiologic and pharmacokinetic changes in pregnancy. Front Pharmacol [Internet]. 2014 Apr 3 [cited 2019 May 23];5:65. Available from: http://www.ncbi.nlm. nih.gov/pubmed/24772083

7. Chandra S, Tripathi AK, Mishra S, Amzarul M, Vaish AK. Physiological Changes in Hematological Parameters During Pregnancy. Indian J Hematol Blood Transfus [Internet]. 2012 Sep 15 [cited 2019 May 23];28(3):144-6. Available from: http://www.ncbi.nlm.nih.gov/pubmed/23997449

8. Akinbami AA, Ajibola SO, Rabiu KA, Adewunmi AA, Dosunmu AO, Adediran A, et al. Hematological profile of normal pregnant women in Lagos, Nigeria. Int J Womens Health [Internet]. 2013 May [cited 2019 May 23];5:227-32. Available from: http://www.dovepress.com/hematologicalprofile-of-normal-pregnant-women-in-lagos-nigeria-peerreviewed-article-IJWH

9. Bakrim S, Motiaa Y, Ouarour A, Masrar A. Hematological parameters of the blood count in a healthy population of pregnant women in the Northwest of Morocco (TetouanM'diq-Fnideq provinces). Pan Afr Med J [Internet]. 2018 Apr 9 [cited 2019 May 23];29:205. Available from: http:// www.panafrican-med-journal.com/content/article/29/205/ full/

10. Gebreweld A, Bekele D, Tsegaye A. Hematological profile of pregnant women at St. Paul's Hospital Millennium Medical College, Addis Ababa, Ethiopia. BMC Hematol [Internet]. 2018 Dec 9 [cited 2019 May 23];18(1):15. Available from https://bmchematol.biomedcentral.com/articles/10.1186/ s12878-018-0111-6

11. Gonzales Rengifo GF, Fano D, Vásquez-Velásquez C. Diagnosis of anemia in populations at high altitudes. Rev Peru Med Exp Salud Publica. 2017;34(4).

12. Dewey KG, Oaks BM. U-shaped curve for risk associated with maternal hemoglobin, iron status, or iron supplementation. Am J Clin Nutr [Internet]. 2017 Dec [cited 2018 Sep 10];106(Supplement 6):1694S-1702S. Available from: http://www.ncbi.nlm.nih.gov/pubmed/29070565

13. Organización Mundial de la Salud. Oficina Regional para las Américas., Organización Panamericana de la Salud. Recomendaciones de la OMS sobre atención prenatal para una experiencia positiva del embarazo [Internet]. Washington; 2016 [cited 2019 Apr 27]. Available from: http:// www.clap.ops-oms.org/publicaciones/9789275320334esp. pdf

14. Steer PJ. Maternal hemoglobin concentration and birth weight. Am J Clin Nutr [Internet]. 2000 May 1 [cited 2018 Sep 10];71(5):1285S-1287S. Available from: http://www. ncbi.nlm.nih.gov/pubmed/10799403

15. Domínguez Ruiz de León P, Morcillo Cebolla V, Gutiérrez Parres B, Cirujano Pita FJ, Díaz de Tuesta Díaz de Lezama E, Mazorra Benito E. Estudio de macrocitosis sin anemia en una población urbana. Atención Primaria [Internet]. 2011 Apr [cited 2019 May 22];43(4):183-9. Available from: http://www.ncbi.nlm.nih.gov/pubmed/20619506

16. Solak $\mathrm{Y}$, Yilmaz MI, Saglam M, Demirbas $S$, Verim $S$, Unal $\mathrm{HU}$, et al. Mean corpuscular volume is associated with endothelial dysfunction and predicts composite cardiovascular events in patients with chronic kidney disease. Nephrology [Internet]. 2013 Nov [cited 2019 Jun 26];18(11):728-35. Available from: http://www.ncbi.nlm. nih.gov/pubmed/23848392

17. Osta V, Segura C, Tissera G, Ayuso C. Estudio de eficiencia y sensibilidad de alarmas de dos analizadores hematológicos en un hospital pediátrico*. Acta Bioquím Clín Latinoam [Internet]. 2014 [cited 2019 Jun 26];48(1):71-80. Available from: http://www.scielo.org.ar/pdf/abcl/v48n1/ v48n1a10.pdf

18. O'brien KO, Ru Y. Iron status of North American pregnant women: an update on longitudinal data and gaps in knowledge from the United States and Canada. Am J Clin Nutr [Internet]. 2017 [cited 2019 Apr 17];106:1647-54. Available from: https://doi.org/10.3945/ajcn.

19. Chabot-Richards DS, George TI. White blood cell counts: reference methodology. Clin Lab Med [Internet]. 2015 Mar [cited 2019 May 20];35(1):11-24. Available from: https:// linkinghub.elsevier.com/retrieve/pii/S0272271214001036

20. Martín Núñez G, Ramos Fernández de Soria R, Fernández Galán MA, Sánchez Gil F, Cuesta P, Martín Borregón $\mathrm{J}$, et al. [Detection campaign for hemoglobinopathies and thalassemias among school children in northern Extremadura]. Sangre (Barc) [Internet]. 1995 Dec [cited 2019 Jun 9];40(6):459-64. Available from: http://www.ncbi. nlm.nih.gov/pubmed/8850228

21. Taylor DJ, Lind T. Haematological changes during normal pregnancy: iron induced macrocytosis. $\mathrm{Br} \mathrm{J}$ Obstet Gynaecol [Internet]. 1976 Oct [cited 2019 Jun 9];83(10):760-7. Available from: http://www.ncbi.nlm.nih. gov/pubmed/990214

22. Solak Mekić M, Pedišić I, Šobat H, Vučićević Boras V, Kirac I, Štefančić L, et al. THE ROLE OF COMPLETE BLOOD COUNT PARAMETERS IN PATIENTS WITH 
COLORECTAL CANCER. Acta Clin Croat [Internet]. 2018 Dec [cited 2019 Jun 9];57(4):624-9. Available from: http:// www.ncbi.nlm.nih.gov/pubmed/31168198

23. The World Bank. Prevalence of anemia among pregnant women (\%) | Data [Internet]. 2016 [cited 2019 Apr 17]. Available from: https://data.worldbank.org/indicator/sh.prg. anem

24. Ministerio de Salud (MINSA). Plan Nacional de Reducción y Control de la Anemia y la Desnutrición Crónica Infantil en el Perú : 2017-2021. Minsa [Internet]. 2016 [cited 2018 Jun 8]; Available from: http://www.minsa.gob.pe/portada/ Especiales/2016/anemia/documentos/000ANEMIA_ PLAN_MINSA_06Feb.pdf

25. Páez M, Cioccia Anna M., Hevia P. Papel de la hepcidina y la ferroportina en la regulación hormonal de la homeostasis del hierro. (Revisión). Vitae Acad Biomédica Digit [Internet].
2014 [cited 2018 Dec 12];59(1). Available from: https:// dialnet.unirioja.es/servlet/articulo?codigo $=6463028$

26. Vásquez-Velásquez C, Aguilar L, López J, Paredes T, Guevara E, Rubín De Celis V, et al. ¿La medición de hemoglobina es más costo-efectiva que el uso del hemograma automatizado? Rev Peru Investig Matern Perinat. 2019; 8(2):27-39.

Correspondencia: Cinthya Vásquez Velásquez Dirección: Av. Honorio Delgado 430. San Martín de Porres. Lima, Perú.

Correo: cinthya.vasquez.v@upch.pe

Teléfono: 01-319-0000 (anexo: 233213) 